\title{
DESIGN OF DIGITAL FILTERS WITH GENERAL HARDWARE CONSTRAINTS BY MEAN FIELD ANNEALING
}

\author{
Per Persson, Sven Nordebo, Ingvar Claesson \\ Department of Telecommunications and Signal Processing \\ University of Karlskrona/Ronneby \\ S-372 25 Ronneby, Sweden \\ per.persson@its.hk-r.se
}

\begin{abstract}
The mean field annealing (MFA) algorithm is presented as an efficient tool to design digital filters with discrete coefficients and hardware constraints. As an application example the algorithm is used to design a finite wordlength digital filter with a simple hardware constraint. The ability of the deterministic MFA algorithm to approximate the mean behavior of the stochastic simulated annealing algorithm is indicated by numerical results.
\end{abstract}

\section{INTRODUCTION}

When designing digital filters of tinite wordlength it would be beneficial if hardware realization aspects could be taken into account and optimized at the same time. Such a codesign process is by its nature a combinatorial problem and very time consuming due to the inherent complexity of combinatorial optimization.

A digital filter design can be considered as a search over a binary-valued state space consisting of all outcomes of $b \times N$ binary variables where $N$ is the number of filter coefficients and $b$ is the coefficient wordlength. The goal is to find the state corresponding to the minimum global cost, consisting of two parts, one associated with the filter specification and the other with the hardware constraints.

Several approaches to this problem have been proposed, among them simulated annealing (SA) and genetic (evolutionary) algorithms $[5,2,10,3]$. Simulated annealing in particular is known to provide good results, but is very timeconsuming due to its stochastic search for thermal equilibrium at each annealing step [1].

The mean field annealing (MFA) algorithm $[4,9,6]$ is a deterministic annealing method capable of handling large combinatorial optimization problems with a minimum of computational effort. It can be regarded as approximating the mean behavior of the simulated annealing algorithm but without the need for a stochastic sampling of states. By applying a technique known as the mean field approximation
[7] the mean values of the decision variables are analytically estimated directly from the Boltzmann distribution instead of iterating a stochastic perturbation rule a large number of times as with SA. The MFA approach proves to be significantly faster and still yields a solution with a quality equal to that of a highly tuned SA algorithm.

\section{PROBLEM FORMULATION}

As a design example for the MFA algorithm we choose an FIR-filter with a minimum number of ones in its binary coding. Such a design is simple enongh not to draw attention from the optimization algorthm while still representing a hard combinatorial problem.

Consider the design of a linear phase nonrecursive (FIR) filter with impulse response $h(n)$, even symmetry $h(n)=$ $h(N-1-n)$, and odd length $N$. The frequency response is given by [8]

$$
\begin{aligned}
& H(\omega)=\sum_{n=0}^{N-1} h(n) \epsilon^{-j \omega n}=\epsilon^{-j \omega M} A(\omega) \\
& A(\omega)=\sum_{m=0}^{M} a_{m} \dot{\partial}_{m}(\omega)=\mathbf{a}^{T} \phi(\omega)
\end{aligned}
$$

where $A(\omega)$ is the gain function, $M=(N-1) / 2$, $a$ is an $(M+1) \times 1$ vector of coefficients $a_{n}=h(M-m)$, and $\phi(\omega)$ is an $(M+1) \times 1$ vector of basis functions $\phi_{m}(\omega)$ where $\phi_{0}(\omega)=1$ and $\phi_{m}(\omega)=2 \cos (m \omega)$ for $m=1, \ldots, M$.

A simple coding of the filter coefficients is the two's complement form

$$
a_{m}=-s_{m b+1}+\sum_{l=1.1}^{b-1} s_{m b+1+1} 2^{-l}
$$

where the new binary variables $\varepsilon_{i} \in\{0,1\}$ have been introduced and $b$ is the coefficient wordlength. Hence, in terms 
of the binary variables the response $A(\omega)$ can be written

$$
A(\omega)=\mathrm{s}^{T} \psi(\omega)
$$

where $s$ is an $L \times 1, L=(M+1) b$ vector of variables $s_{i}$, $\psi(\omega)=\phi(\omega) \propto \mathrm{d}$ and $\mathrm{d}=\left[-12^{-1} \ldots 2^{-b+1}\right]^{T}$.

We consider the following quadratic design criterion whose cost function is defined by

$$
E(\mathrm{~s})=\underbrace{\sum_{k=1}^{K} u_{k}^{2}\left(A\left(\omega_{k}\right)-A_{d}\left(\omega_{k}\right)\right)^{2}}_{\text {filter design cost }}+\alpha \underbrace{\sum_{i=1}^{L} s_{i}^{2}}_{\text {constraint }}
$$

where $A_{d}\left(\omega_{k}\right)$ is the desired response defined on a set of frequency points $w_{k} \in[0, \pi]$ for $k=1, \ldots, K, w_{k}$ is a stopband weighting and $\alpha$ is a positive parameter used to trade off the filter design cost (first tern above) for the hardware cost defined in terms of the total number of binary ones (second term above). The choice of cost function is not critical to the algorithm and e.g. a minimax filter specification with the hardware constraints given by the maximum hamming distance would be treated similarly.

By employing (4) we may now rewrite the cost function in (5) as the following quadratic form

$$
E(\mathbf{s})=\mathrm{s}^{T}(\mathbf{R}+\alpha \cdot \mathbf{I}) \mathrm{s}-2 \mathrm{p}^{T} \mathrm{~s}+c
$$

where

$$
\begin{aligned}
& \mathrm{R}=\sum_{k=1}^{K} w_{k}^{2} \psi\left(\omega_{k}\right) \psi^{T}\left(\omega_{k}\right) \\
& \mathrm{p}=\sum_{k=1}^{K} w_{k}^{2} \psi\left(\omega_{k}\right) A_{d}\left(\omega_{k}\right) \\
& c=\sum_{k=1}^{K} w_{k}^{2} A_{d}^{2}\left(\omega_{k}\right) .
\end{aligned}
$$

We emphasize that the type 1 linear phase FIR filter structure $[8]$ and the quadratic design criterion chosen above are used merely as an example to demonstrate the potential of the new algorithm; other filter structures, design criteria and hardware costs may be treated similarly.

\section{THE MEAN FIELD ANNEALING ALGORITHM}

By mapping the filter design problem and the corresponding hardware constraints onto the set of binary variables, $s_{i}$, it can be solved using a mean field annealing (MFA) technique. Consider the set of states $S$ defined by

$$
S=\left\{s \mid s_{i} \in\{0,1\}, i=1, \ldots, L\right\}
$$

and the global cost, $E(\mathrm{~s})$, connected with each state. From statistical mechanics it is well known that for a system in thermal equilibrium at temperature $T$ the probability of a particular state $s$ is given by the Boltzmann distribution

$$
P(s)=\frac{e^{-E(s) / T}}{\sum_{s} e^{-E(s) / T}}
$$

and that the expectation value of variable $s_{i}$, denoted by $\left\langle s_{i}\right\rangle$, will be

$$
\left\langle s_{i}\right\rangle_{T}=\frac{\sum_{S} s_{i} e^{-E(s) / T}}{\sum_{S} e^{-E(s) / T}} .
$$

Under the condition that the system is kept in thermal equilibrium, it is easy to show [1] that (11) gives

$$
\lim _{T \rightarrow 0} P\left(s^{o p t}\right)=1
$$

where $\mathrm{s}^{o p t}$ is the state with $E\left(\mathrm{~s}^{o p t}\right)<E\left(\mathrm{~s} \neq \mathrm{s}^{\text {opt }}\right)$.

For most problems (12) is impossible to compute in a reasonable anount of time but a good approximation known as the mean field approximation $[6,7]$ is derived by rewriting (12) using conditional expectations over $s_{i}=\{0,1\}$ as

$$
\left\langle s_{i}\right\rangle_{T}=\frac{\sum_{S} \epsilon^{-E(s) / T^{2}} \frac{\sum_{s_{i}=0,1} s_{i} e^{-\frac{l}{T} E(s) s_{i}}}{\sum_{s_{i}=0,1} e^{-\frac{1}{T} E(\mathrm{~s}) s_{i}}}}{\sum_{S} \epsilon^{-E(\mathrm{~s}) / T}} .
$$

Now, (14) can be expressed as

$$
\left\langle\theta_{i}\right\rangle_{T}=\frac{\sum S e^{-E(\mathrm{~s}) / T} \frac{1}{1+e^{\Delta E_{i}(B) / T}}}{\sum_{S} e^{-E(\mathrm{~s}) / T}}
$$

where

$$
\Delta E_{i}(\mathbf{s})=\left.E(\mathbf{s})\right|_{s_{i}=1}-\left.E(\mathbf{s})\right|_{s_{i}=0}
$$

and the following identity is established:

$$
\left\langle\theta_{i}\right\rangle_{T}=\left\langle\frac{1}{1+e^{\Delta E_{i}(\mathrm{~s}) / T}}\right\rangle_{T} .
$$

The approximation consists of moving the expectation operator $\langle\cdot\rangle$ in the right hand side of (17) inside the argument of $\Delta E_{i}(\cdot)$,

$$
\left(s_{i}\right)_{T} \approx \frac{1}{1+\epsilon^{\Delta E_{i}\left(s_{i}\right) / T}} .
$$

By introducing the set of continuous variables $\nu=\{\mathrm{v} \mid$ $\left.v_{i} \in[0,1], i=1, \ldots, L\right\}$ where $v_{i}=\langle s\rangle$, the approximation in (18) can be formulated as a continnous-valued fixed point equation

$$
v_{i}=\frac{1}{1+e^{\Delta E_{i}(\mathbf{v}) / T}}
$$

and the corresponding cost function to minimize becomes

$$
E(\mathbf{v})=\mathbf{v}^{T}(\mathbf{R}+\alpha \cdot \mathbf{I}) \mathbf{v}-2 \mathrm{p}^{T} \mathbf{v}+c
$$




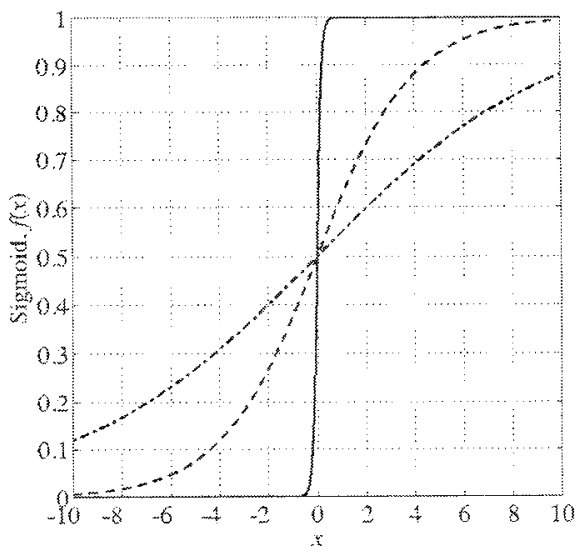

Figure 1: The signoid function $f(x)=1 /\left(1+\epsilon^{-x / T}\right)$ plotted for $T=5$ (dash-dotted), $T^{\circ}=2$ (dashed) and $T=$ 0.1 (solid).

The shape of the sigmoid function (19) for various temperatures is shown in Fig. 1.

Starting from a high value of $T$ and iterating the update rule (19) while decreasing $T$ will accomplish two things. For infinite $T$ the 1rivial fixed point is $v_{i}=0.5$, but as $T \rightarrow 0, v_{i} \rightarrow\{0,1\}$. At the same time the properties of the Boltzmann distribution as $T \rightarrow 0$ (13) ensure that a state with a cost close to the global minimum is reached at $T \approx 0$.

The MFA algorithm can be described as

1 Set $v_{i}=0.5$ and set initial temperature, $T$

2 Calculate $v_{i}$ from (19)

3 Repeat step 2 until $c_{i}$ are stable

4 Decrease the temperature geometrically

5 Repeat steps $2-4$ until all $v_{i}$ are close to $\{0,1\}$

\section{NUMERICAL EXAMPLES}

The MFA-algorithm was used to design an $N=17$ tap linear phase lowpass FIR-filter with coefficients quantized to $b=8$ bits which was then compared to a filter designed straightforwardly by quantizing the coefficients of the infinite precision $L S$-solution, referred to as $Q L S$ below. The weighting parameter $w_{k}$ was set to 1 in the passband and 2 in the stopband. The tradeoff between filter characteristics and hardware constraints is imposed in a soft manner using $\alpha \geq 0$.
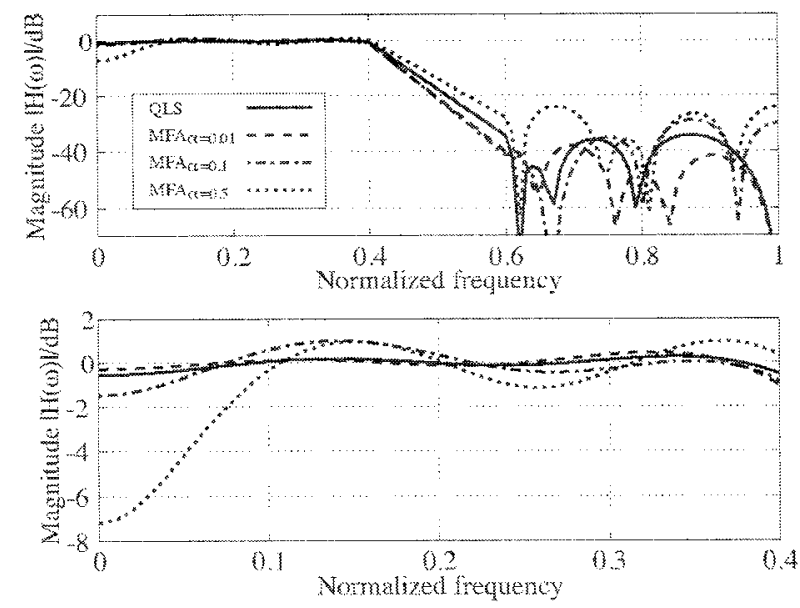

Figure 2: The magnitude response of the filters. The quantized LS-solution (solid) and the MFA-solutions for $\alpha=$ 0.01 (dashed), $\alpha=0.1$ (dash-dotted) and $\alpha=0.5$ (dotted) are drawn. The lower plot shows the passband only.

The results with respect to the quality of the filter defined by $E_{m i n}=\left.E(\mathrm{~s})\right|_{\alpha=0}$ and the corresponding number of ones used in the implementation are listed in Table 1 and the magnitude response of the filters are shown in Fig. 2 .

\begin{tabular}{c|c|c|c} 
& $\alpha$ & no of $1 \mathrm{~s}$ & $E_{m i n}$ \\
\hline \hline QLS & & 34 & 0.0520 \\
\hline MFA & 0.01 & 32 & 0.0492 \\
MFA & 0.10 & 27 & 0.2929 \\
MFA & 0.50 & 21 & 2.1338 \\
\hline
\end{tabular}

Table 1: Comparison between the quantized LS-solution and the MFA-solutions with their respective hardware cost expressed as the number of ones needed.

Note that the response of the MFA-solution with $\alpha=$ 0.01 (dashed) is better than the one obtained simply by quantizing the LS-solution. When $\alpha=0.5$ (dotted) the hardware constraints start degrading the filter performance.

The validity of the MFA-approximation employed in (18) and the use of continuous variables to track the mean behavior of a corresponding simulated annealing procedure are illustrated in Fig. 3 where both SA and MFA were applied to the same problem of size $N=7$ and $b=8$. It is clear that the behavior of $v_{i}$ closely follows that of $\left\langle s_{i}\right\rangle$, 
as was expected. Thus, instead of the time-consuming process of obtaining thermal equilibrium by a stochastic search, the mean field approximation provides thermal equilibriun conditions throngh a deterministic fixed point equation.
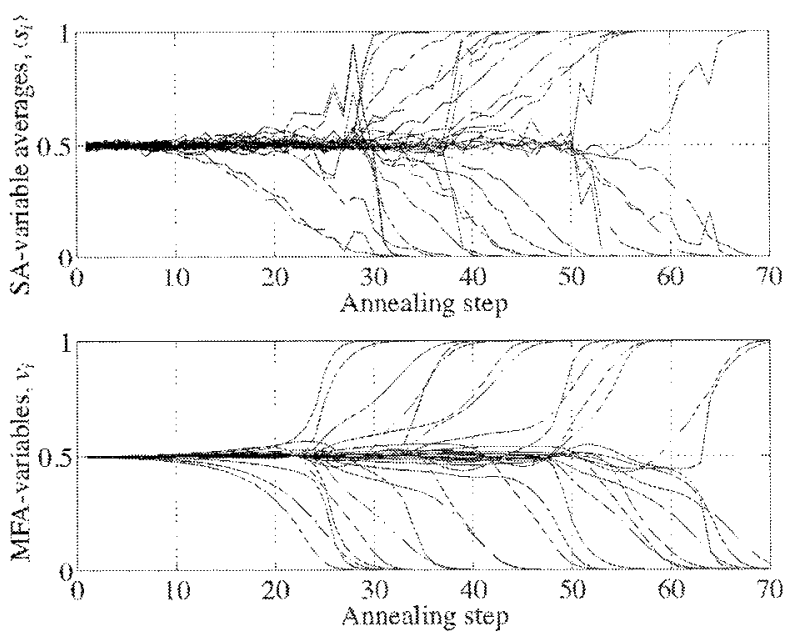

Figure 3: A comparison of the convergence properties of SA and MFA. The upper plot shows how $\left\langle s_{i}\right\rangle$, computed by SA, evolve over the annealing course while the lower plot shows $v_{i}$ when computed by MFA. At each annealing step each $s_{i}$ was iterated 400 times while each $t ;$ on average needed less than 2 iterations to converge.

\section{SUMMARY AND CONCLUSIONS}

The mean field annealing (MFA) algorithm is presented as a means for designing digital filters with hardware constraints. The MFA approach is viewed as an analytical analogy to the well known simulated annealing algorithm and MFA turns out to be less time-consuming while still rendering high quality solutions. Examples are given for a simple linear phase FIR-filter with increasing demands on the hardware aspect. The tradeoff between filter characteristics and hardware constraints is governed by a single parameter, $\alpha$.

The strength of the MFA algorithm in digital filter design is the fact that it operates directly on the bitlevel of the filter, which means that hardware constraints can be smoothly incorporated in the design, and that it does away with the stochastic sampling of states needed in e.g. sinulated annealing.

Even though the examples given are for linear phase FIR-filters the algorithm is clearly not limited to such cases. It is our opinion that it can be turned into a useful tool to design general digital filters, with or without hardware constraints, for which no general method exists today.

\section{REFERENCES}

[1] E. Aarts, J. Korst, Simulated Annealing and Boltzmann Machines. John Wiley \& Sons, Inc., 1989.

[2] N. Benvenuto, M. Marchesi. A. Uncini,"Applications of Simulated Annealing for the Design of Special Digital Filters", IEEE Trans. Signal Processing , vol. 40 , no. 2, pp. 323-332, Feb. 1992.

[3] O. Franzen, H. Blume, H. Scröder, "FIR-filter design with spatial and frequency design constraints using evolution strategies", Signal Processing, vol. 68, pp. $295-306,1998$

[4] J. J. Hopfield, D. W. Tank, "Neural Computation of Decisions in Optimization Problems", Biol. Cybern, vol. 52,pp. 141-152, 1985.

[5] R. V. Kacelanga, P. J. Grauman, L. E. Turner,"Design of Digital Filters Using Simulated Annealing", Proc. IEEE Int. Symp. Circuits Syst. (New Orleans, LA), May 1990, pp. 642645 .

[6] M. Ohlsson, C. Peterson, B. Söterberg, "Neural networks for optimization problems with inequality constraints: The knapsack problem", Neural Computation, vol. 5, no. 2, pp. 331.339, March 1993.

[7] G. Parisi, Statistical Field Theory, Perseus Books Publishing, L.L.C., 1998.

[8] T. W. Parks, C. S. Burrus, Digital Fitter Design, John Wiley \& Sons, Inc., 1987.

[9] C. Peterson, B. Söderberg, "A New Method for Mapping Optimization Problems onto Neural Networks", International Journal of Neural Systems, vol. I, no. 1, pp. 3-22, Jan. 1989.

[10] J. Radecki, J. Konrad, E. Dubois, "Design of multidimensional finite-wordlength FIR and IIR filters by simulated annealing** IEEE Trans. on Circuits and Systems II, vol. 42, no.6. pp. 424431 , June 1995. 